\title{
Fast in vivo multiphoton microscopy using optimized light-sheet illumination
}

\author{
Vincent Maioli, Antoine Boniface, Pierre Mahou, Júlia Ferrer Ortas, \\ Lamiae Abdeladim, Emmanuel Beaurepaire, and Willy Supatto* \\ Laboratory for Optics and Biosciences, Ecole Polytechnique, CNRS, INSERM, IP Paris, \\ 91128 Palaiseau Cedex, France
}

\begin{abstract}
Light-sheet fluorescence microscopy is a method of choice for multiscale live imaging. Indeed, its orthogonal geometry results in high acquisition speed, large field-of-view and low photodamage. Its combination with multiphoton excited fluorescence improves its imaging depth in biological tissues. However, it appears femtosecond laser sources commonly used in multiphoton microscopy at an $80 \mathrm{MHz}$ repetition rate may not be optimized to take full advantage of light-sheet illumination during live imaging. Hence, we investigated the nature of induced photodamage in multiphoton light-sheet microscopy and the influence of laser parameters on the signal-to-photodamage ratio. To this end, we used zebrafish embryonic heart beat rate and fluorophore photobleaching as sensitive reporters of photoperturbations. We characterized linear and nonlinear disruptions depending on laser parameters such as laser mean power, pulse frequency or wavelength, and determine their order and relative impact. We found an optimal pulse frequency of $\sim 10 \mathrm{MHz}$ for imaging mCherry labeled beating hearts at $1030 \mathrm{~nm}$ excitation wavelength. Thus, we achieved high-speed imaging without inducing additional linear heating or reaching nonlinear photodamage compared to previous implementation. We reach an order-ofmagnitude enhancement in two-photon excited fluorescence signal by optimizing the laser pulse frequency while maintaining low both the laser average power and its peak irradiance. It is possible to reach even larger enhancement of 3photon excited fluorescence using such laser parameters. More generally, using low laser pulse frequency in multiphoton light-sheet microscopy results in a drastic improvement in signal level without compromising live sample, which opens new opportunities for fast in vivo imaging.
\end{abstract}

Keywords: nonlinear microscopy, in vivo imaging, single-plane illumination microscopy, heart, zebrafish, two-photon excited fluorescence, three-photon excited fluorescence, 2P-SPIM

\section{INTRODUCTION}

Improving the acquisition speed of multiphoton microscopy for fast in vivo imaging is both required and challenging. Indeed, it requires developing strategies, which do not compromise viability or normal behavior of live sample. Lightsheet illumination has been used to increase the speed of multiphoton microscopy using different nonlinear contrast mechanisms, such as two- or three- photon excited fluorescence ${ }^{1,2}$, or second harmonic generation ${ }^{3}$. Among other strategies for fast acquisition rates in multiphoton microscopy, light-sheet illumination with an orthogonal configuration of illumination and detection provides unique advantages for live imaging with low photodamage ${ }^{4}$. Indeed, compared to other techniques, the illumination parallelization and a good axial resolution are obtained with lower laser mean power and peak intensity. Indeed, since the illumination parallelization is performed in the laser propagation direction, it is obtained using a single weakly focused beam. It then requires lower laser mean power than other parallelization methods, such as multifocal point-scanning or wide-field illumination. In addition, the orthogonal geometry provides similar axial resolution using one order of magnitude lower illumination numerical aperture than in other approaches. Together, such illumination should result in lower linear absorption and thermal effect, as well in reduced highly nonlinear photodamage ${ }^{4}$. However, the laser parameters governing linear and nonlinear photodamage in multiphoton light-sheet microscopy remain poorly investigated. Importantly, we anticipated that the laser parameters commonly used in point-scanning multiphoton microscopy, are not optimal for multiphoton light-sheet microscopy. We hypothesized that characterizing the nature and relative contribution of photoperturbations involved in multiphoton light-sheet microscopy would result in a specific and efficient strategy to improve signal while maintaining low photodisruption.

*willy.supatto@polytechnique.edu 


\section{RESULTS}

The work presented here aimed at optimizing the laser illumination parameters, such as mean power and pulse frequency, to find the best balance between nonlinear signal and photodamage during live imaging with multiphoton light-sheet microscopy. Compared to other strategies developed for fast multiphoton imaging, multiphoton light-sheet microscopy holds a unique position in terms of photodamage, which is due to its orthogonal arrangement of illumination and detection. Indeed, other approaches such as fast scanning, multifocal or wide field illumination are based on a collinear arrangement, which results in five inportant differences: (i) low illumination focusing of a single beam, (ii) high pulse energy limit for fluorescence saturation, (iii) low peak intensity, (iv) low mean power, and (v) long pixel dwell times.

(i) Firstly, the orthogonal geometry allows us to perform a parallelization of the illumination by using weakly focused beam (or a low illumination numerical aperture, NA) without compromising spatial resolution or signal. For instance, wide field illumination in the collinear geometry results in loss of axial resolution, which can be circumvented by using temporal focusing. This is not required in orthogonal illumination due to the inherent optical sectioning. In our case, we used an illumination of 0.09 NA to obtain an axial resolution $\sim 3 \mu \mathrm{m}^{5}$.

(ii) Secondly, the weakly focused beam results in high saturation levels of fluorophores. Indeed, as shown in previous work $^{6}$, the pulse energy limit for fluorescence saturation $\mathrm{E}_{\text {sat }}$ scales as $\mathrm{NA}^{-2}$. In our experimental conditions, $\mathrm{E}_{\text {sat }}$ of mCherry typically increases from $\sim 1$ to $>100 \mathrm{~nJ}$ when comparing collinear and orthogonal approaches (Table 1 ). We then used Charan et $\mathrm{al}^{6}$ estimation of the optimum repetition rate $f_{0}$ limited by fluorescence saturation at a specific imaging depth $\mathrm{z}$ (relative to the tissue attenuation length $l_{a}$ ) as presented in Table 1. It confirms high saturation values enable us to use significantly lower laser repetition rate than $80 \mathrm{MHz}$ without reaching fluorophore saturation. In practice, we performed live imaging using an energy per pulse at the sample $\mathrm{E}_{\text {pulse }}<10 \mathrm{~nJ}$ which is well below $\mathrm{E}_{\text {sat }}$ in the case of a weakly focused beam (Table 1). In our case, the optimal repetition rate is not limited by fluorophore saturation, but by nonlinear photodamage. By contrast, other fast multiphoton imaging strategies using a collinear geometry (for instance, fast scanning $^{7}$ or multifocal ${ }^{8}$ approaches), involved $\mathrm{E}_{\text {pulse }}$ close or higher than the fluorophore saturation level.

\begin{tabular}{|c|c|c|c|c|c|c|c|c|c|c|}
\hline Dye & $\begin{array}{c}\text { Cross-section } \\
\text { in GM }\end{array}$ & $\begin{array}{c}\lambda \\
\text { in nm }\end{array}$ & NA & $\begin{array}{c}\tau \\
\text { in fs }\end{array}$ & $\begin{array}{c}\text { Esat } \\
\text { in nJ }\end{array}$ & \multicolumn{5}{|c|}{$f_{0}$ in MHz } \\
\hline & & & & & & $\mathrm{z}=l_{a}$ & $\mathrm{z}=2 l_{a}$ & $\mathrm{z}=3 l_{a}$ & $\mathrm{z}=4 l_{a}$ & $\mathrm{z}=5 l_{a}$ \\
\hline eGFP & $\sim 40$ & 940 & 1.0 & 100 & $\mathbf{0 . 3 7}$ & 335 & 123 & 45.3 & 16.7 & 6.1 \\
\hline eGFP & $\sim 40$ & 940 & 0.09 & $\sim 300$ & $\mathbf{7 8}$ & 1.57 & 0.58 & 0.21 & 0.08 & 0.03 \\
\hline mCherry & $\sim 10$ & 1030 & 1.0 & 100 & $\mathbf{0 . 8 0}$ & 153 & 56.2 & 20.7 & 7.6 & 2.8 \\
\hline mCherry & $\sim 10$ & 1030 & 0.09 & $\sim 300$ & $\mathbf{1 7 2}$ & 0.71 & 0.26 & 0.10 & 0.04 & 0.01 \\
\hline
\end{tabular}

Table 1. Saturation pulse energy $E_{\text {sat }}$ and optimal repetition rate $f_{0}$ limited by saturation in multiphoton light-sheet microscopy. Calculation assumptions are based on previous work ${ }^{6}$ in the case mCherry and eGFP fluorophores. The last line in green corresponds to parameters used in this study. $\lambda$ : excitation wavelength; NA: illumination numerical aperture; $\tau$ : laser pulse width.

(iii) In addition, the low illumination NA results in significantly lower peak intensities. We typically used $0.1 \mathrm{TW} . \mathrm{cm}^{-2}$ at the sample. Other fast multiphoton techniques using tightly focused beams typically require one to two order of magnitude stronger peak intensities ${ }^{7,8}$, which promotes nonlinear photodamage.

(iv) Furthermore, the orthogonal geometry allows parallelizing the illumination with a single beam. Considering the signal is nonlinear, splitting the beam such as with multifocal illumination is less efficient and requires using higher mean powers to reach sufficient signal levels ${ }^{4}$. In the case of light-sheet illumination, the same photons from a single beam illuminate several points within the imaging plane. As a result, only $<100 \mathrm{~mW}$ is required for fast imaging, which is typically at least one order of magnitude lower than in the case of multifocal ${ }^{8}$ or wide field illumination ${ }^{9}$. Using higher mean power can lead to linear photoperturbation, such as heating.

(v) Finally, the parallelization of the illumination and detection results in long illumination and collection time per pixel while maintaining high pixel and frame rate. In our case, we used $76 \mu$ s illumination time per pixel to image at 168 frames per second and $40 \mathrm{MHz}$ pixel rate with multiphoton light-sheet microscopy. By contrast, fast multiphoton imaging using collinear illumination is often achieved with extremely short pixel dwell times (2 to $400 \mathrm{~ns}$ in recent reports ${ }^{7,8}$ ). The long dwell time involved in multiphoton light-sheet microscopy allows us to use low laser repetition rate without limiting the imaging speed. Indeed, unlike in point-scanning techniques, a long delay between pulses does not limit the frame rate. At $f=10 \mathrm{MHz}$ laser repetition rate, each pixel in our recorded images was still illuminated by 760 pulses. 
Together, these specific properties of multiphoton light-sheet microscopy, which result from its orthogonal geometry, call for different optimization parameters to mitigate photodamage. To this end, we devised a systematic experimental workflow and investigated the nature and level of photodamage induced during imaging of live zebrafish embryos. We specifically investigated the effect of the laser repetition rate $f=1 / T$. As expected, we experimentally verified that the 2 and 3-photon excited fluorescence signals increase with $\mathrm{T}$ at a constant mean power in a linear or quadratic manner, respectively (Fig. 1a-b). However, this signal enhancement is limited by potential nonlinear photodamage due to high peak intensities. To characterize the threshold of photodamage, we then used heartbeat rate as a sensitive reporter of linear and nonlinear disruptions ${ }^{5}$. By analyzing 500 experimental conditions in 100 embryos, we established the scaling law governing highly nonlinear photodamage (Fig. 1c). In addition, we quantified linear effects such as heating due to water absorption and photobleaching ${ }^{5}$. Together, these analyses established the signal enhancement limit, which is reachable by adjusting the laser repetition rate $\mathrm{f}=1 / \mathrm{T}$ at a given mean power (Fig. 2). It demonstrates that the limiting parameters differ from the ones previously reported using point-scanning multiphoton microscopy ${ }^{10}, 11$ and call for a different optimization strategy. Indeed, at $f=80 \mathrm{MHz}$ repetition rate, we demonstrated that photoperturbations are not limited by highly nonlinear photodamage, photobleaching or fluorophore saturation but by linear absorption and heating. In the case of two-photon excite fluorescence (2PEF, Fig. 2a), signal enhancement can then be obtained by decreasing the laser repetition rate while maintaining a constant mean power. As a result, by adjusting the laser pulse frequency, we obtained a $\sim$-fold increase in two-photon excited fluorescence signal without inducing additional linear effects or reaching nonlinear photodamage (Fig. 3). Similar improvement is obtained with second harmonic generation ${ }^{5}$. Due to its higher order, even stronger enhancement ( 64) is reached with three-photon excited fluorescence (Fig. 2b). To illustrate such optimization of signal-to-photodamage ratio, we imaged the zebrafish beating heart at a $40 \mathrm{MHz}$ pixel rate (Fig. 3), with improved signal-to-noise ratio compared to previous report ${ }^{2}$. We then achieved high-speed multiphoton imaging in vivo while maintaining both low laser average power $(70 \mathrm{~mW})$ and peak irradiance $\left(0.1 \mathrm{TW} . \mathrm{cm}^{-2}\right)$. Indeed, the laser power was at least one order of magnitude lower than used in other parallelized techniques using multifocal point-scanning or wide-field illumination. In addition, the laser peak irradiance was maintained below typical values used in standard multiphoton point-scanning microscopy.

a) Two-photon excited fluorescence

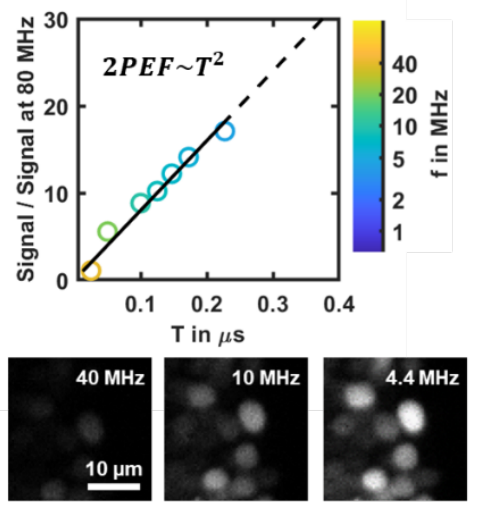

b) Three-photon excited fluorescence

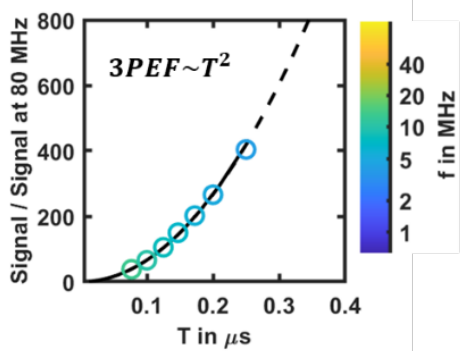

$\mathrm{f}$ in $\mathrm{MHz}$

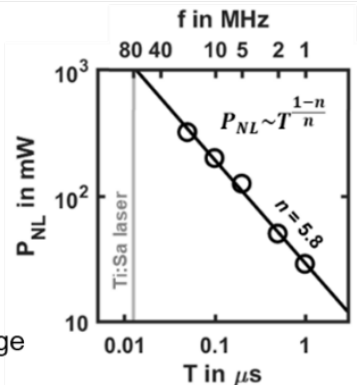

Figure 1. Scaling laws governing signal and nonlinear photodamage in multiphoton light-sheet microscopy microscopy. (a) Two-photon excited fluorescence (2PEF) linearly depends on laser period $\mathrm{T}=1 / \mathrm{f}$ (a) when imaging mCherry labeled nuclei in zebrafish embryos (see image insets at the bottom). (b) Three-photon excited fluorescence (3PEF) quadratically depends on laser period $\mathrm{T}=1 / \mathrm{f}$ when imaging blue fluosphreres. (c) Scaling law of nonlinear photodamage induced during multiphoton light-sheet imaging of the zebrafish beating heart. The mean power threshold $\left(\mathrm{P}_{\mathrm{NL}}\right)$ of nonlinear photodamage is plotted depending on the laser period $\mathrm{T}=1 / \mathrm{f}$. It corresponds to a highly nonlinear process of order $\mathrm{n}=5.8$. 

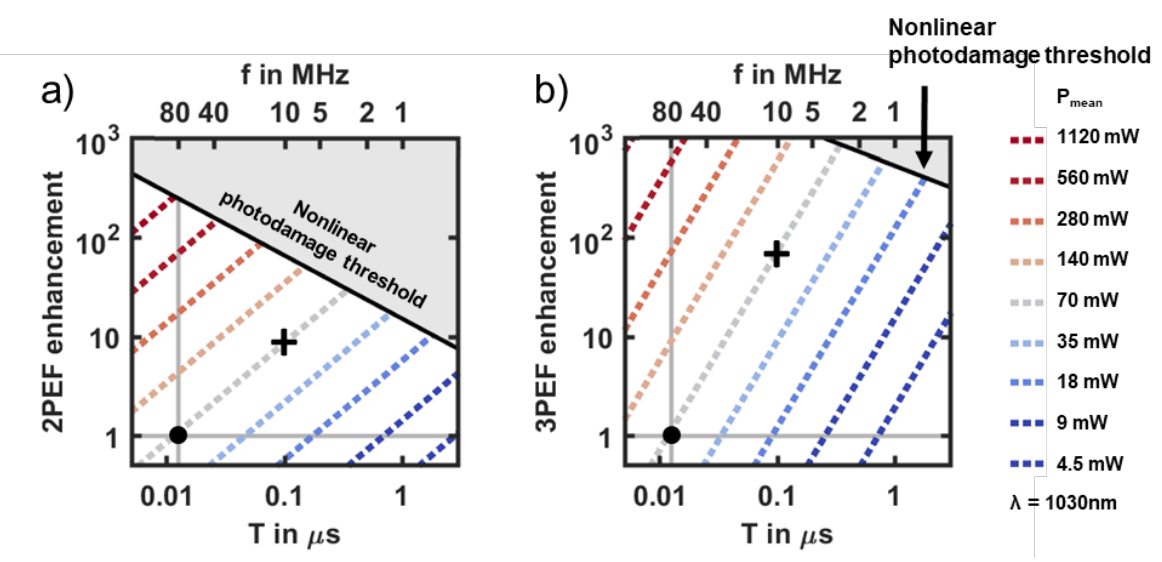

Figure 2. Signal enhancement limited by nonlinear photodamage threshold in multiphoton light-sheet microscopy. (a) When using twophoton-excited fluorescence (2PEF) to image mCherry labeled nuclei in the zebrafish beating heart at 1030nm excitation wavelength, the signal enhancement is compared to standard illumination parameters (black dot, at $70 \mathrm{~mW}$ mean power and $80 \mathrm{MHz}$ repetition rate). Black cross, imaging conditions used in Fig. 3. at $10 \mathrm{MHz}$ laser repetition rate. (b) Higher enhancement can be obtained using threephoton-excited fluorescence (3PEF) while staying far from nonlinear photodamage threshold. Solid black lines correspond to the nonlinear photodamage threshold estimated by experiments. Dashed blue-to-red lines corresponds to line of constant mean power and constant linear effect (linear variation of heart beat rate and of temperature).

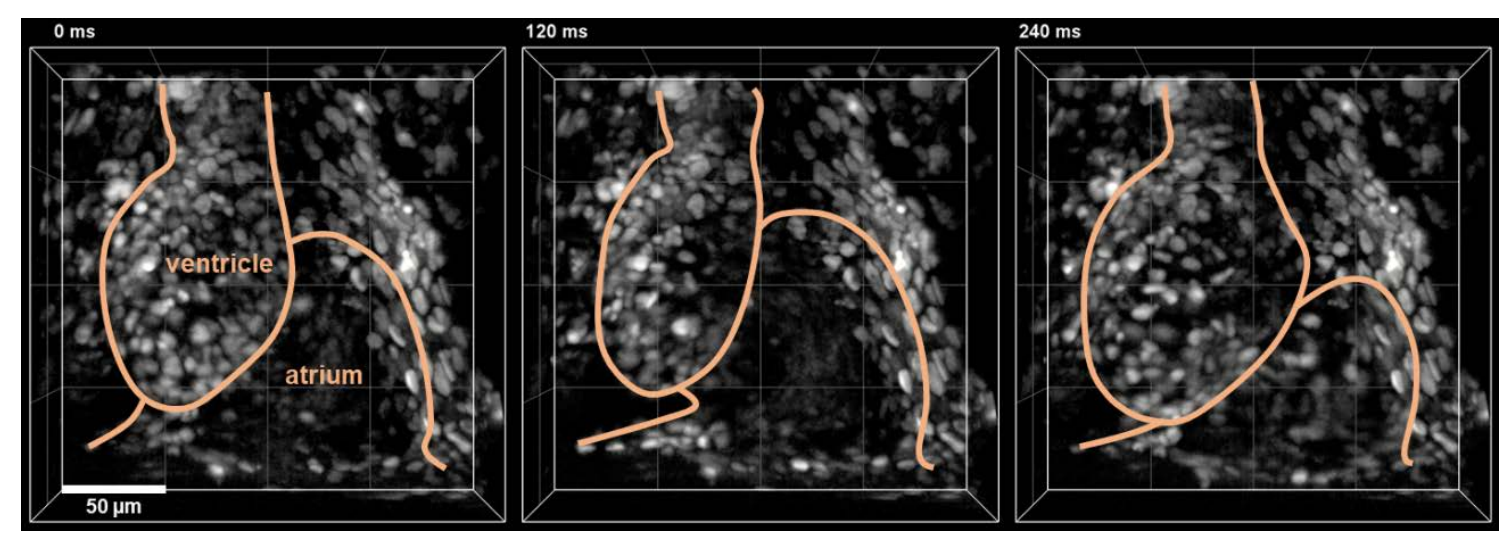

Figure 3. Fast imaging of the zebrafish heart using two-photon light-sheet microscopy at optimized laser pulse frequency. Time series of images acquired at 168 frames per second using $\mathrm{f}=10 \mathrm{MHz}$ repetition rate, a $70 \mathrm{~mW}$ mean power, and $1030 \mathrm{~nm}$ wavelength. Ubiquitous mCherry labeling of cell nuclei. Heart outlined in orange.

\section{CONCLUSION}

Orthogonal illumination in multiphoton light-sheet microscopy entails a different photodamage regime compared to other high-speed multiphoton strategies. Using an experimental approach, we explored the specific balance between signal and linear or nonlinear photodamage in multiphoton light-sheet microscopy. We demonstrated that commonly-used pulsed lasers at a repetition rate of $80 \mathrm{MHz}$ were not optimal to take full advantage of light-sheet illumination. We have shown that an increase of one order of magnitude in the level of two-photon excited fluorescence signal can be gained by using lower repetition rate without reaching nonlinear photodamage and by keeping linear heating constant. Even stronger enhancement is reachable in the case of three-photon excited fluorescence. Finally, we demonstrated high-speed multiphoton imaging (typically $40 \mathrm{MHz}$ pixel rate) of the beating heart in zebrafish embryos with low mean power, low peak intensity and long pixel dwell time. Such significant improvement in signal levels opens new opportunities for fast in vivo imaging with multiphoton light-sheet microscopy. 


\section{REFERENCES}

[1] Truong, T. V., Supatto, W., Koos, D. S., Choi, J. M., and Fraser, S. E., “Deep and fast live imaging with twophoton scanned light-sheet microscopy,” Nat. Methods, 8(9), 757-760 (2011).

[2] Mahou, P., Vermot, J., Beaurepaire, E., and Supatto, W., “Multicolor two-photon light-sheet microscopy,” Nat. Methods, 11(6), 600-1 (2014).

[3] Malkinson, G., Mahou, P., Chaudan, É., Gacoin, T., Sonay, A. Y., Pantazis, P., Beaurepaire, E., and Supatto, W., "Fast In Vivo Imaging of SHG Nanoprobes with Multiphoton Light-Sheet Microscopy,” ACS Photonics, 7(4), 1036-1049 (2020).

[4] Supatto, W., Truong, T. V., Débarre, D., and Beaurepaire, E., “Advances in multiphoton microscopy for imaging embryos,” Curr. Opin. Genet. Dev., 21(5), 538-48 (2011).

[5] Maioli, V., Boniface, A., Mahou, P., Ortas, J. F., Abdeladim, L., Beaurepaire, E., and Supatto, W., "Fast in vivo multiphoton light-sheet microscopy with optimal pulse frequency,” Biomedical Optics Express, 11(10), 6012-6026 (2020).

[6] Charan, K., Li, B., Wang, M., Lin, C. P., and Xu, C., "Fiber-based tunable repetition rate source for deep tissue two-photon fluorescence microscopy,” Biomed. Opt. Express, 9(5), 2304-2311 (2018).

[7] Wu, J., Liang, Y., Chen, S., Hsu, C. L., Chavarha, M., Evans, S. W., Shi, D., Lin, M. Z., Tsia, K. K., and Ji, N., "Kilohertz two-photon fluorescence microscopy imaging of neural activity in vivo,” Nat. Methods, 17(3), 287-290 (2020).

[8] Zhang, T., Hernandez, O., Chrapkiewicz, R., Shai, A., Wagner, M. J., Zhang, Y., Wu, C. H., Li, J. Z., Inoue, M., Gong, Y., Ahanonu, B., Zeng, H., Bito, H., and Schnitzer, M. J., "Kilohertz two-photon brain imaging in awake mice,” Nat. Methods, 16(11), 1119-1122 (2019).

[9] Amor, R., McDonald, A., Tragardh, J., Robb, G., Wilson, L., Abdul Rahman, N. Z., Dempster, J., Amos, W. B., Bushell, T. J., and McConnell, G., "Widefield Two-Photon Excitation without Scanning: Live Cell Microscopy with High Time Resolution and Low Photo-Bleaching,” PLoS One, 11(1), e0147115 (2016).

[10] Ji, N., Magee, J. C., and Betzig, E., "High-speed, low-photodamage nonlinear imaging using passive pulse splitters,” Nat. Methods, 5(2), 197-202 (2008).

[11] Debarre, D., Olivier, N., Supatto, W., and Beaurepaire, E., "Mitigating phototoxicity during multiphoton microscopy of live Drosophila embryos in the 1.0-1.2 microm wavelength range,” PLoS One, 9(8), e104250 (2014). 